\title{
Implantação do prontuário eletrônico do paciente no Brasil
}

\author{
Luciano Garcia Lourenção, D.Sc.*, Cleber de Jesus Ferreira Junior, M.Sc.**
}

**Enfermeiro, Faculdade de Medicina de São José do Rio Preto (FAMERP), **Dentista, Departamento de Gestão em Informática em Saúde, Escola Paulista de Medicina (EPM), Universidade Federal de São Paulo (UNIFESP)

\begin{abstract}
Resumo
Objetivo: Conhecer as percepções sobre a implantação do Prontuário Eletrônico do Paciente (PEP) no Brasil. Método: Realizou-se busca bibliográfica na Biblioteca Virtual em Saúde, através da Bireme, nas Bases Eletrônicas Medline, Lilacs, Scielo e pelo endereço eletrônico scholar.google.com.br, utilizando-se os descritores: registros eletrônicos de saúde, sistemas computadorizados de registros médicos, sistemas de registro de ordens médicas, continuidade da assistência ao paciente, registros médicos. Resultados: As principais temáticas das publicaçôes estudadas foram: implantação e utilização do PEP, criação de ambiente para visualizaçáo de imagens médicas, utilização de agentes móveis para atualização do PEP, modelo de PEP para Estratégia Saúde da Família e avaliação da utilização do PEP. Conclusão: Embora haja dificuldades, o uso da informática traz avanços ao sistema de saúde, sendo necessárias novas pesquisas para a consolidação da implantação do PEP e a melhoria da assistência à saúde da população.
\end{abstract}

Palavras-chave: registros eletrônicos de saúde, sistemas computadorizados de registros médicos, sistemas de registro de ordens médicas, continuidade da assistência ao paciente, registros médicos.

\section{Abstract \\ Implementation of patient electronic health record in Brazil}

Objective: To know the perceptions regarding implementation of patient electronic health record (EHR) in Brazil. Method: Literature search was held in the Virtual Health Library, through Bireme, Electronic Bases Medline, Scielo and Lilacs by the electronic address scholar.google.com.br, using the key-words: electronic health records, computerized medical records systems, registry systems orders, continuity of patient care, medical records. Results: The main themes of the studied publications were: implementation and use of EHR, creation of environment for viewing medical images, use of mobile agents for updating the EHR, EHR model for the Family Health Strategy and evaluation of the use of EHR. Conclusion: Although there are difficulties in using this program, information technology brings advances to health system. Thus, new researches to consolidate the implementation of the EHR and improvement of health care of the population are needed.

Key-words: electronic health record, computerized medical records systems, medical order entry systems, continuity of patient care, medical records.

Recebido em 16 de outubro de 2015; aceito em 30 de outubro de 2015. Endereço para correspondência: Luciano Garcia Lourenção, Faculdade de Medicina de São José do Rio, Departamento de Epidemiologia e Saúde Coletiva, Avenida Brigadeiro Faria Lima, 5416, 15090-000 São José do Rio Preto SP, E-mail: luciano.famerp@gmail.com 


\section{Resumen}

\section{Implementación del registro electrónico de salud del paciente en Brasil}

Objetivo: Conocer las percepciones acerca de la implementación del Registro de Pacientes Electrónico (RPE) en Brasil. Métodos: Se ha realizado una búsqueda bibliográfica en la Biblioteca Virtual en Salud, a través de la Bireme, en las Bases Electrónicas Medline, Lilacs, Scielo y por la dirección electrónica scholar.google.com.br, utilizando los descriptores: registros electrónicos de salud, sistemas de registros médicos computadorizados, sistemas de entrada de órdenes médicas, continuidad de la atención al paciente, registros médicos. Resultados: Los principales temas de publicaciones estudiados fueron: implantación y uso de RPE, creación de ambiente para la visualización de imágenes médicas, el uso de agentes móviles para actualizar el RPE, modelo RPE para la Estrategia de Salud de la Familia y la evaluación del uso de la RPE. Conclusión: Aunque hay dificultades, el uso de tecnologías de la información trae avances de sistema de salud, siendo necesarias nuevas investigaciones para consolidar la aplicación de la RPE y la mejora de la salud de la población.

Palabras-clave: registros electrónicos de salud, sistemas de registros médicos computadorizados, sistemas de entrada de órdenes médicas, continuidad de la atención al paciente, registros médicos.

\section{Introdução}

O termo prontuário é derivado do latim prontuarium, o qual se relaciona a um livro de anotações devidamente classificadas e organizadas em certa ordem, dispostas em tópicos que tem como objetivo facilitar o diagnóstico. Entretanto, sabe-se que o prontuário de papel adotado predominantemente pelos serviços de saúde até os dias atuais não absorve totalmente o seu conceito. Dessa forma, o Prontuário Eletrônico do Paciente (PEP) surge como um novo modelo de tratamento da informação em saúde, apresentando-se como instrumento auxiliar nas ações de atenção à população, independente do local que o sujeito se encontre e do profissional que promova os cuidados $[1,2]$.

Para Possari [3], o PEP é objeto e processo, pois descreve e registra toda a cadeia de eventos, desde a inserçáo do paciente no sistema até os serviços médicos prestados, que incluem os procedimentos, prescriçóes e exames realizados. Tendo como proposta básica reunir os diferentes tipos de informações relativas ao estado de saúde e à assistência prestada ao paciente, durante todo seu ciclo vital, tem potencial para representar uma fonte de informaçóes fundamental no processo de tomada de decisão.

Considerando que os PEPs possuem uma série de funcionalidades que contemplam o registro de informaçôes do paciente; informaçóes clínicas; registros de atendimentos médicos e de enfermagem e muitas outras, podemos ver que essa ferramenta abarca uma série de tarefas que se enquadram em diferentes âmbitos - tanto na assistência direta ao paciente quanto na gestão [4].

Como benefícios do uso de sistemas de PEP, para a assistência à saúde, temos: maior disponibilidade e acesso mais amplo a informaçóes de saúde, as quais estão mais atualizadas e legíveis; alertas em casos de inconsistências e interaçôes medicamentosas, aumentando a segurança do paciente; possibilidade de comparar computacionalmente resultados de exames e a evolução do tratamento, auxiliando o diagnóstico e o plano assistencial; capacidade de rápido compartilhamento de informações, quando necessário; geração de relatórios e indicadores de gestão e assistenciais, tanto para fins epidemiológicos quanto estatísticos, colaborando para os processos de acreditação, entre outros [5].

Destaca-se, ainda, que os sistemas de PEP permitem controle da segurança e auditoria, resultando em maior privacidade para todos os envolvidos. Os sistemas computacionais em muito podem ajudar os profissionais em seus procedimentos clínicos. Ao mesmo tempo fazem um registro claro e preciso do ato clínico, impossibilitando alteraçóes, e identificando univocamente o profissional, ficando a responsabilização mais evidente. Essa característica resulta em uma assistência mais atenciosa e consciente, consequentemente de melhor qualidade [5].

Ante o exposto, este estudo tem o objetivo de conhecer as percepçóes sobre a implantação do Prontuário Eletrônico do Paciente (PEP) no Brasil, a partir de diferentes experiências e identificar quais foram os resultados obtidos nestas experiências. 


\section{Métodologia}

Trata-se de um estudo de revisão bibliográfica sobre percepçóes e resultados em experiências de implantação do Prontuário Eletrônica do Paciente (PEP) no Brasil, ou relacionada a esta temática, publicadas a partir da década de 2000, com análise de conteúdo do assunto enfocado.

Segundo Cervo e Bervian [6], a pesquisa bibliográfica busca explicar um problema a partir de referências teóricas publicadas em documentos. Pode ser realizada independente ou como parte da pesquisa descritiva ou experimental.

Para a realização da pesquisa bibliográfica, Gil [7] propóe quatro leituras: a primeira exploratória, realizada de forma rápida, com o objetivo de considerar ou não interessante a publicação para o estudo; a segunda determina quais materiais bibliográficos são interessantes para o estudo em questão; a terceira é analítica, com o ordenamento das informaçôes adquiridas de forma a responder os objetivos da pesquisa e a quarta é a leitura interpretativa, sendo identificados os significados obtidos por meio da leitura analítica, que tem por objetivo relacionar o que o autor afirma com o problema para o qual se propóe uma solução.

Lakatos e Marconi [8] ressaltam que a leitura interpretativa relaciona as afirmaçóes do autor com os problemas para os quais quem estuda ou pesquisa busca uma solução. Trata-se de uma associaçáo de ideias, pelas quais se seleciona apenas o que é pertinente e útil, o que contribui para resolver o problema proposto.

A fonte de busca das informaçóes científicas foi a Biblioteca Virtual em Saúde (BVS), por meio da Bireme (Centro Latino-Americano e do Caribe de Informação em Ciências da Saúde), nas Bases Eletrônicas Medline (Medical Literature Analysis and Retrietal System On-Line), Lilacs (Literatura Latino-Americana e do Caribe em Ciências da Saúde), Scielo (Scientific Eletronic Library On Line) e Google (Scholar Google). Os descritores utilizados foram: registros eletrônicos de saúde, sistemas computadorizados de registros médicos, sistemas de registro de ordens médicas, continuidade da assistência ao paciente, registros médicos.

A partir do levantamento bibliográfico foi realizado o ordenamento e análise da bibliografia científica selecionada, de modo a avaliar e discutir os principais aspectos apresentados nos estudos com relação à temática "implantação do Prontuário
Eletrônico do Paciente (PEP) no Brasil", considerando a distribuição das publicaçóes segundo estado de origem, o período de publicaçáo e, em cada publicação, a fonte e título, o foco de estudo e as principais conclusóes.

\section{Resultados}

Foram selecionadas e analisadas 14 publicaçôes sobre implantação do Prontuário Eletrônica do Paciente (PEP) no Brasil, ou relacionada a esta temática, sendo $11(78,57 \%)$ resumos de anais de congressos, $1(7,14 \%)$ dissertaçáo de mestrado e 2 $(14,29 \%)$ artigos.

A Tabela I apresenta a distribuição das publicaçóes segundo regiāo de origem dos autores, verificando-se uma maior prevalência de estudos sobre esta temática nas regióes Sudeste $(64,29 \%)$ e Sul $(28,57 \%)$ do Brasil.

Tabela I - Distribuição das publicações segundo a região de origem dos autores. São José do Rio Preto, 2015.

\begin{tabular}{lcc}
\hline \multicolumn{1}{c}{ REGIÃO } & N $^{\circ}$ & $\%$ \\
\hline Sul & 4 & 28,57 \\
Sudeste & 9 & 64,29 \\
Centro-Oeste & 0 & 0 \\
Norte & 1 & 7,14 \\
Nordeste & 0 & 0 \\
\hline TOTAL & 14 & 100 \\
\hline
\end{tabular}

Em relação ao ano de publicação, foram selecionadas publicaçóes a partir do ano 2000. Observou-se um maior número de publicaçóes no período de 2000 a 2006, com diminuiçáo da produçấo científica sobre a temática, a partir de 2007, conforme demonstrado na Tabela II.

Tabela II - Distribuição das publicações segundo o período e ano de publicação. São José do Rio Preto, 2015.

\begin{tabular}{lcc}
\hline \multicolumn{1}{c}{ Período/Ano de Publicação } & $\mathrm{N}^{\circ}$ & $\%$ \\
\hline 2000 a 2006 & 11 & 78,57 \\
\hline 2000 & 1 & 7,14 \\
2002 & 3 & 21,43 \\
2004 & 1 & 7,14 \\
2005 & 1 & 7,14 \\
2006 & 5 & 35,71 \\
\hline 2007 a 2014 & 3 & 21,43 \\
\hline
\end{tabular}




\begin{tabular}{lcc}
\hline 2007 & 1 & 7,14 \\
2012 & 1 & 7,14 \\
2013 & 1 & 7,14 \\
\hline TOTAL & 14 & 100 \\
\hline
\end{tabular}

A Tabela III apresenta a distribuição das publicaçōes segundo autores/título, fonte, foco do estudo e recomendaçóes/conclusóes. Observou-se que as publicaçóes analisadas abordavam temáticas como implantaçáo e utilizaçáo do PEP, criação de ambiente para visualização de imagens médicas, utilização de agentes móveis para atualização do PEP, modelo de PEP para Estratégia Saúde da Família e Avaliação da utilização do PEP.

Os estudos analisados mostram conclusóes/ recomendaçóes importantes como: há impactos ne- gativos do PEP sobre o trabalho dos profissionais; os profissionais acreditam que o PEP contribui para melhorar a qualidade na assistência prestada ao indivíduo; as informaçóes do PEP são utilizadas principalmente para atender as questóes de cunho administrativo; apesar de desvantagens do PEP, os profissionais optariam pelo seu uso; muitos profissionais ainda têm dúvidas sobre o funcionamento do PEP e suas potencialidades; há carência de informaçóes sobre o PEP para a Estratégia Saúde da Família no Brasil, sendo necessário incentivar a criação e avaliação contínua de modelos de prontuários de família; os profissionais recomendam a expansão do PEP para os serviços de saúde e, aqueles que o utilizam, não querem voltar para processos manuais.

Tabela III - Distribuição das publicações segundo autores/título da publicação, fonte, foco do estudo e conclusões/ recomendações. São José do Rio Preto, 2015.

\begin{tabular}{ccc}
\hline $\begin{array}{c}\text { Autores/título da publi- } \\
\text { cação }\end{array}$ & Fonte & Foco do estudo \\
\hline
\end{tabular}

Furuie S, Rebelo $M$,

Gutierrez M, Moreno R, Anais do Nardon F, Motta G, et al. [9]

Prontuário eletrônico em ambiente distribuído e heterogêneo: a experiência do InCor. CBIS'2002 VIII Congresso Brasileiro de Informática em Saúde, 2002.

\section{Criação de} ambiente para visualização de imagens médicas.
Conclusões / recomendações
O estudo discute todas as etapas da implantação de um ambiente distribuído e heterogêneo para visualização de imagens médicas no InCor. Foram utilizados padrões abertos em todas as etapas do desenvolvimento do projeto, permitindo flexibilidade e robustez a todos os modelos e implementações realizados.

O modelo e a infraestrutura construídos são amplos e permitem a representação e manipulação de qualquer tipo de dado ou documento médico. O servidor DICOM desenvolvido em Java neste projeto é atualmente utilizado para armazenamento e transmissão de imagens do PACS-InCor e o sistema de controle de acesso é utilizado para acesso ao Prontuário Eletrônico.

O estudo mostrou que há impactos negativos do PEP sobre o trabalho dos profissionais, como o aumento do tempo de atendimento e o possível comprometimento da relação médico-paciente, porém os profissionais acreditam que o PEP melhora o acesso e a qualidade das informações, organiza as tarefas e facilita o monitoramento do paciente, contribuindo para melhorar a qualidade na assistência prestada ao indivíduo.

Os autores pretendem incentivar novas pesquisas sobre o assunto, que possam enriquecer ainda mais os estudos sobre esta ferramenta, de forma a amenizar seus impactos negativos e aumentar os impactos positivos, permitindo aos profissionais e às instituições de saúde tirar proveito de todos os seus benefícios e melhorar a assistência prestada à saúde da população. 
Charnovscki R,

Nascimento Filho JR, Machado GB, Nicoleit ER [ 11 1]

Registro eletrônico para acompanhamento médico de pacientes em uma UTI.
IX Congresso Brasileiro de Informática em

Saúde. Ribeirão Preto, 2004.
Implantação do PEP.

A primeira versão do sistema foi avaliada pelos profissionais que solicitaram uma versão mais funcional. A nova versão será provida de relatórios mais detalhados que os gerados atualmente e um módulo para análises estatísticas.

Equipe médica e de enfermagem têm contribuído no desenvolvimento e avaliação do sistema, destacando a legibilidade das informações, a automatização de alguns processos e a facilidade na recuperação de dados como principais vantagens.

O banco de dados atende à necessidade de recuperação de informações de forma ágil e segura.

O PEP gerou ganho no conteúdo das informações e redução de custo no manuseio de prontuários em papel, além de agilizar o acesso às informações.

Nunes DCC, Cotta Junior A, Lima MOSS [12] prontuário eletrônico do paciente no Hospital Márcio Cunha - 6 anos de sucesso.
XVIII Congresso

Brasileiro de Informática em Saúde, 2006. Florianópolis/

SC.
Implantação do

PEP.

Para que o hospital tenha um prontuário exclusivamente eletrônico é necessário que ações conjuntas sejam realizadas entre os hospitais, fornecedores de soluções e outras entidades envolvidas no processo de certificação de software, para culminar num PEP que atenda aos requisitos de segurança, conteúdo e funcionalidades de um Sistema de Registro

Eletrônico em Saúde - SRES.

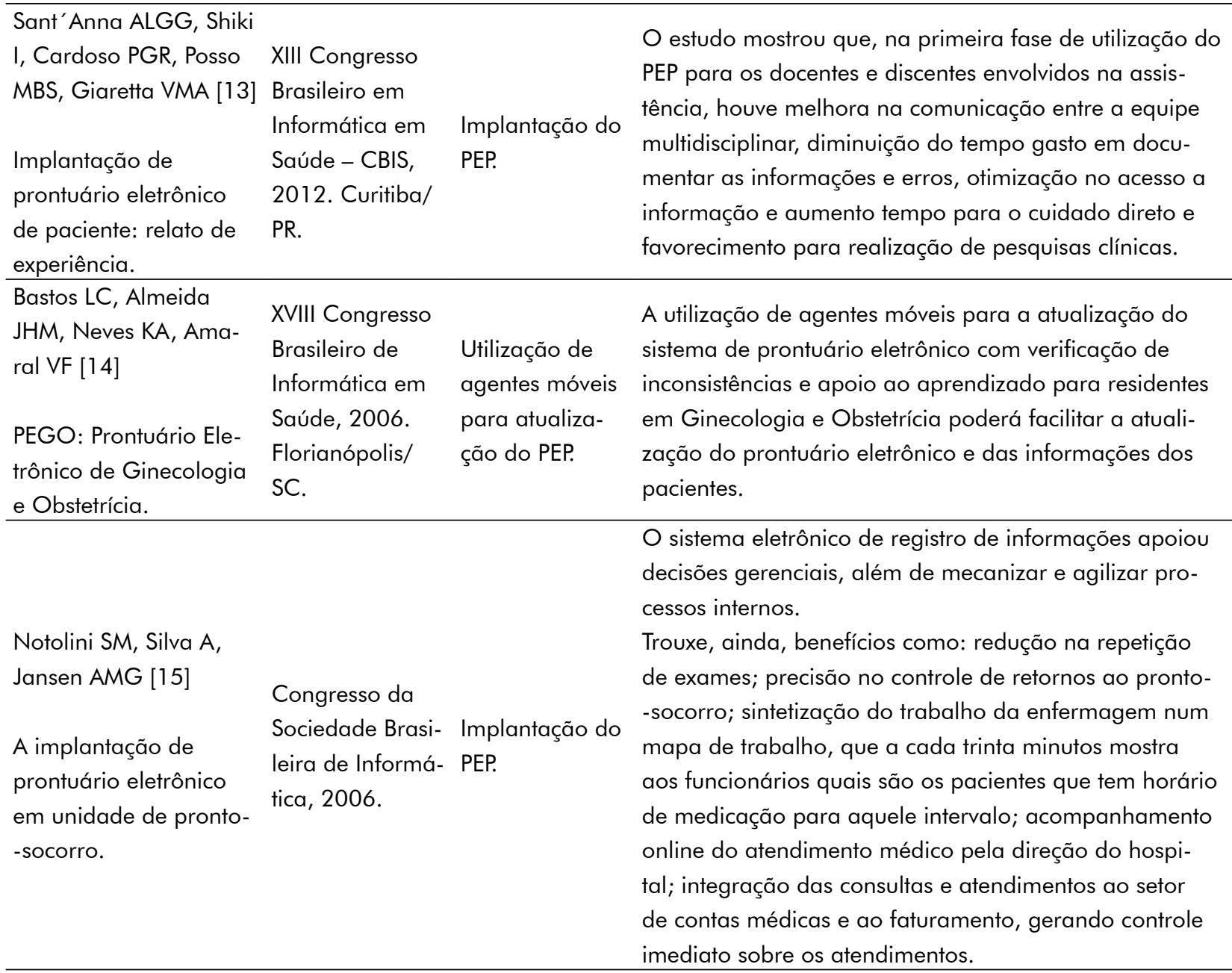


Negreiros MM, Tavares-

-Neto J [16]

Proposta de prontuário

para as equipes de

Saúde da Família.
Revista APS

2005;8(2): 123 -

42.
Modelo de PEP para Estratégia Saúde da Família.

O estudo analisou prontuários de Programas de Residência Médica (PRM) de Medicina de Família e Comunidade (MFC) do Brasil e de países da América Latina e do Canadá, para propor modelo de prontuário para a Estratégia Saúde da Família (ESF). Foram analisados 3 prontuários brasileiros e 7 estrangeiros. $O$ modelo proposto foi dividido em três partes: caracterização da família; indicadores socioeconômicos e dados pessoais dos moradores de cada domicílio. Os resultados do estudo evidenciaram a carência dessas informações no Brasil e a necessidade de fomento visando a criação e avaliação contínua de modelos de prontuários de família.

$\mathrm{O}$ autor investigou o primeiro ano de funcionamento do PEP e seus antecedentes em um hospital de alta complexidade, para entender as mudanças decorrentes da

Programa de

Murahovsci D [17]

Pós-Graduação da AESPIFGV.

Implantação de prontuário eletrônico em um hospital de grande porte: estudo de caso.

Área de Concen- Implantação do tração: Adminis- PEP. tração Hospita-

lar e de Sistemas de Saúde. implantação do prontuário eletrônico no hospital e examinar resistências ao processo. Concluiu que a extensão e o grau em que o prontuário eletrônico afeta o hospital como um todo, foi maior do que a suposição inicial. Constatou-se que a informática tem o poder de evidenciar problemas há muito ocultos na estrutura hospitalar, tais como: prontuários que fornecem informações incompletas e frequentemente ilegíveis, falhas no processo de suprimento de materiais e medicamentos, rotinas hospitalares inadequadas que se perpetuam.

$O$ estudo verificou que a maioria dos profissionais utiliza

Namorato L, Neto AJC, Garani FV, Braga PO, Lustosa SAS [18]

A utilização do prontuário eletrônico do paciente por médicos do Hospital Municipal Dr. Munir Rafful: um estudo de caso.
$\mathrm{J}$ Health Inform 2013;5(2):3943. Utilização do PEP. o PEP, principalmente, em hospitais ou consultórios particulares. A principal vantagem apontada pelos médicos foi a facilidade de leitura e as falhas no sistema a principal desvantagem. Apesar das desvantagens apontadas, a maioria dos médicos optaria pelo PEP. Para os autores, o funcionamento do PEP e suas potencialidades ainda são fonte de dúvidas para a maior parte dos médicos do hospital estudado. $O$ estudo demonstra a necessidade da disseminação e discussão de informações a respeito do PEP junto aos profissionais. 
Rodrigues LC, Souza

TM, Barbon Jr. S, Lucat-

to Junior RV [19]

\section{Gerenciamento de} prontuário eletrônico de pacientes em clínica de atendimento fisioterápico do Centro Universitário do Norte Paulista.
XVIII Congresso

Brasileiro de Informática em

Implantação do PEP. Saúde, 2006.

O estudo apresenta um PEP que agrupa as informações exigidas por modelos médicos padrões, além de acessar e exibir todas as imagens relacionadas a determinado paciente, podendo ser examinadas junto com seu histórico e outras informações relacionadas com o paciente, uma vez que estas estejam alimentadas no banco de dados responsável pelo armazenamento de todas as informações do sistema. Aborda, ainda, o módulo de consulta, onde algumas opções para consulta e filtragens estão disponíveis a médicos e outros profissionais. A partir da experiência inicial, propõe a continuidade na implementação do PEP, efetivando o controle da evolução do mesmo e eliminando a duplicidade dos dados nos diversos centros de atendimento, através da incorporação de todos os prontuários de atendimento em um mesmo sistema. Posteriormente, pretende-se implementar módulos de mineração de dados e padrões para troca de informações entre diversos sistemas, independentemente da plataforma de hardware e software utilizadas.

No processo de implantação do PEP Odontológico em Curitiba foram identificados fatores limitantes relacionados ao processo de trabalho da odontologia, como espaço físico e biossegurança.

Contudo, o processo de informatização na Secretaria Municipal de Saúde de Curitiba tem contribuído para a melhoria da qualidade da gestão em saúde, uma vez que é um instrumento de racionalização do trabaIho, otimização dos custos e melhoria da qualidade do atendimento, sendo, portanto, uma ferramenta gerencial importante. Permite, ainda, melhorias na gestão clínica odontológica, na medida em que está previsto um módulo com parâmetros de monitoramento da qualidade do serviço.

O PEP Odontológico foi concebido considerando os diferentes pontos de atenção e as especificidades dos Programas: Saúde Bucal, Saúde da Família, Amigo Especial, Diagnóstico Bucal, Atenção ao Idoso, Ortodontia Preventiva, Endodontia e Periodontia. 


\begin{tabular}{|c|c|c|}
\hline \multicolumn{3}{|l|}{ Sancho LG, Rievrs N, } \\
\hline Sena E [21] & Anais do X Con- & \\
\hline Avaliação do Projeto & & Avaliação da \\
\hline Gestão Saúde em & de Intormática & utilização do \\
\hline Rede: um primeiro & 2006 Florianó- & PEP. \\
\hline olhar do período pós & polis/SC. & \\
\hline $\begin{array}{l}\text { informatização, na } \\
\text { perspectiva da gestão. }\end{array}$ & & \\
\hline
\end{tabular}

Santos AF, Trad R, Santos SF, Dornas Júnior G, Anais do XVIII Costa RB, Ribeiro CA, Congresso Ruas SSM [22] Brasileiro de Engenharia Avaliação da implantação do Prontuário Biomédica Eletrônico na Secretaria Municipal de Saúde de SBIS. São José dos Campos, UniVap, 2002. Belo Horizonte.
O estudo mostra que as informações são utilizadas principalmente para atender as questões de cunho administrativo em detrimento das relativas à apreensão do objeto de trabalho e dos produtos do trabalho. Ou seja, são pouco utilizadas para o planejamento das ações relativas à atenção à saúde e melhora na qualidade da atenção, mas sim para atender a melhora na organização do fluxo do serviço.

Os resultados apontam que o sistema deve, à luz da sua proposição, ser flexibilizado e estar em contínuo processo de (re)especificação, com o objetivo de responder principalmente ao uso da informação à luz da gestão. O estudo avalia o impacto da utilização do prontuário eletrônico em 14 unidades básicas de saúde da Secretaria Municipal de Saúde de Belo Horizonte. Os resultados demonstram que, para a maioria dos profissionais ocorreu melhoria na organização de tarefas da unidade, facilidades para o monitoramento de pacientes e clareza quanto às informações registradas. Contudo, a avaliação quanto ao tempo necessário para o registro das informações difere - 43,5\% avaliam que aumentou e $52 \%$ avaliam que diminuiu. Os profissionais pesquisados recomendam a expansão do projeto para outras unidades e não querem voltar para processos manuais.

ma de saúde brasileiro. Dentre os aspectos positivos da implantação do PEP, destacam-se:

- melhoria do acesso e da qualidade das informaçóes, organizaçáo das tarefas e facilidades no monitoramento do paciente, contribuindo para melhoria da qualidade na assistência prestada ao indivíduo [10];

- há ganhos no conteúdo das informaçóes e redução de custo no manuseio de prontuários em papel, além de agilizar o acesso às informaçóes [12];

- melhoria na comunicação entre a equipe multidisciplinar, diminuição do tempo gasto em documentar as informaçóes e erros, otimização no acesso a informação e aumento tempo para o cuidado direto e favorecimento para realização de pesquisas clínicas [13];

- redução na repetição de exames, precisão no controle de retornos ao pronto-socorro; sintetização do trabalho da enfermagem num mapa de trabalho que, a cada trinta minutos, mostra aos funcionários quais são os pacientes que tem horário de medicação para aquele intervalo; acompanhamento online do atendimento médico pela direção do hospital; integração das consultas e atendimentos ao setor de contas médicas e ao faturamento, gerando controle imediato sobre os atendimentos [15]; 
- melhoria da qualidade da gestáo em saúde, pois racionaliza o trabalho, otimiza os custos e melhora a qualidade do atendimento [20].

Por outro lado, algumas dificuldades são apontadas, a partir das experiências relatadas, as quais representam fragilidades a serem superadas, a partir do aprimoramento do sistema, da capacitação profissional e de investimentos em tecnologia que garantam avanços no processo de implantaçáo do PEP no Brasil. Entre os aspectos negativos apresentados pelos autores estudados, destacam-se:

- aumento do tempo de atendimento e o possível comprometimento da relação médico-paciente [10];

- falta de padróes de PEP para utilizaçáo em serviços específicos, vigentes em todo o território nacional, como a Estratégia Saúde da Família [16];

- subutilização das informaçóes para o planejamento das ações relativas à atenção à saúde e melhora na qualidade da atençáo, privilegiando o uso das mesmas para atender a melhora na organização do fluxo do serviço, ou seja, para atender as questóes de cunho administrativo [21].

Em termos gerais, o uso da informática nos serviços de saúde representa grandes avanços, apesar de todas as dificuldades que possa abarcá-la. Para Murahovsci [17], o uso da informática permite evidenciar problemas ocultos na estrutura hospitalar, tais como: prontuários que fornecem informaçóes incompletas e frequentemente ilegíveis, falhas no processo de suprimento de materiais e medicamentos, além de rotinas hospitalares inadequadas que se perpetuam.

Portanto, se considerarmos que a implantação e uso dos PEPs implicam no uso da tecnologia e apresentam diversas funcionalidades que contribuem tanto para o processo assistencial quanto para o gerencial, esse sistema pode contribuir de forma significativa para a melhoria dos serviços de saúde [4].

Para Santos et al. [22], a introdução de tecnologias de informação é percebida de forma positiva por trabalhadores dos serviços de saúde, trazendo importantes avanços na organizaçáo do processo assistencial, propriamente dito. Corroborando estes autores, Charnovscki et al. [11] destacam que a participação dos profissionais médicos e de enfermagem no processo de melhorias do sistema de registro eletrônico é fundamental para o apri- moramento do mesmo. Ao discorrerem sobre o registro eletrônico para acompanhamento médico em UTI, estes autores destacam como principais vantagens do sistema, a legibilidade das informaçóes, a automatização de alguns processos e a facilidade na recuperação de dados.

Por outro lado, como já elencado, as fragilidades estão presentes, afinal, estamos falando de um processo ainda em desenvolvimento no Brasil e, considerando as diferenças de infraestrutura dos serviços, a necessidade de altos investimentos em tecnologia e o preparo profissional para uso dessa ferramenta, nem sempre as experiências de implantação do PEP são exitosas.

Contudo, profissionais acreditam que o PEP melhora o acesso e a qualidade das informaçóes, organiza as tarefas e facilita o monitoramento do paciente, contribuindo para melhorar a qualidade na assistência prestada ao indivíduo [10].

Namorato et al. [18] destacam que o maior uso do PEP ocorre em hospitais ou consultórios particulares, embora, de maneira geral, a maioria dos profissionais utilizam o PEP, quando implantado. Segundo os autores, médicos apontam a facilidade de leitura como principal vantagem apontada e as falhas no sistema como a principal desvantagem. Apesar destas desvantagens, a maioria dos médicos optaria pelo PEP. Os autores afirmam que a maioria dos médicos possuem dúvidas sobre o funcionamento do PEP e suas potencialidades, evidenciando a necessidade da disseminação e discussão de informaçôes a respeito do PEP junto aos profissionais.

Para Mouráo e Neves [10], é importante que sejam estimuladas novas pesquisas sobre o PEP, consolidando o conhecimento sobre esta ferramenta, amenizando os impactos negativos e fortalecendo os aspectos positivos, para garantir aos profissionais e às instituiçóes de saúde aproveitar todos benefícios possíveis do PEP, contribuindo para a melhoria do sistema de saúde e da assistência prestada à saúde da população.

\section{Conclusão}

$\mathrm{Na}$ análise da bibliografia sobre a implantação do PEP no Brasil, observou-se que esta ferramenta tem sido implementada em diversos segmentos dos serviços de saúde, havendo prevalência de aspectos positivos sobre os negativos, no processo de implantação. 
Embora o PEP ainda apresente fragilidades, os profissionais optariam pelo uso desta ferramenta, recomendando sua expansão nos serviços de saúde.

Portanto, conclui-se que, apesar das dificuldades inerentes ao processo de implantação do PEP nos serviços de saúde, o uso da informática representa grandes avanços ao sistema. Contudo, novas pesquisas são necessárias, para o avanço desta ferramenta e o processo de sua implantaçáo, garantindo melhorias para o sistema de saúde brasileiro e na assistência oferecida à populaçáo.

\section{Referências}

1. Stumpf MK, Freitas HMR. A gestão da informação em um hospital universitário: em busca da definiçáo do patient core record. Rev Adm Contemp 1997;1(1).

2. Mourão AD, Neves JTR. Impactos da Implantação do Prontuário Eletrônico do Paciente sobre o Trabalho dos Profissionais de Saúde da Prefeitura Municipal de Belo Horizonte. Simpósio de Excelência em Gestão e Tecnologia - SEGET; 2007.

3. Possari JF. Prontuário do paciente e os registros de enfermagem. São Paulo: Iátria; 2005.

4. Marin HF, Miranda CF. Prontuário Eletrônico do Paciente. [Apostila]. Universidade Federal de São Paulo, Departamento de Informática em Saúde. São Paulo: Unifesp; 2014.

5. Saúde Business School. Conheça as funcionalidades de um Prontuário Eletrônico. 30 de julho de 2012. [citado 2014 Jul 18]. Disponível em URL: http://saudeweb. com.br/31592/conheca-as-funcionalidades-de-um-prontuario-eletronico

6. Cervo AL, Bervian PA. Metodologia científica. 5a ed. São Paulo: Pearson; 2002.

7. Gil AC. Como elaborar projetos de pesquisa. 3a ed. São Paulo: Atlas; 1996. 175p.

8. Lakatos EM, Marconi MA. Fundamentos de metodologia científica. 6a. ed. São Paulo: Atlas; 2005.

9. Mourão AD, Neves JTR. Impactos da implantação do prontuário eletrônico do paciente sobre o trabalho dos profissionais de saúde da Prefeitura Municipal de Belo Horizonte. Belo Horizonte: Faculdade Cenecista de Varginha - FACECA; 2007.

10. Furuie S, Rebelo M, Gutierrez M, Moreno R, Nardon F, Motta G, et al. Prontuário eletrônico em ambiente distribuído e heterogêneo: a experiência do InCor. In: VIII Congresso Brasileiro de Informática em Saúde; 2002.

11. Charnovscki R, Nascimento J, Machado G, Nicoleit E. Registro eletrônico para acompanhamento médico de pacientes em uma UTI. In: IX Congresso Brasileiro de Informática em Saúde. Ribeirão Preto, 2004. Disponível em URL: http://www.sbis.org.br/cbis9/ arquivos/650.pdf.

12. Nunes DCC, Cotta Junior A, Lima MOSS. O prontuário eletrônico do paciente no hospital Márcio Cunha -6 anos de sucesso. In: XVIII Congresso Brasileiro de Informática em Saúde, Florianópolis SC; 2006.

13. Sant'Anna ALGG, Shiki I, Cardoso PGR, Posso MBS, Giaretta VMA. Implantação de Prontuário Eletrônico de Paciente: relato de experiência. In: XIII Congresso Brasileiro em Informática em Saúde - CBIS; 2012.

14. Bastos LC, Almeida JHM, Neves KA, Amaral VF. PEGO: Prontuário eletrônico de ginecologia e obstetrícia. In: XVIII Congresso Brasileiro de Informática em Saúde; 2006.

15. Notolini SM, Silva A, Jansen AMG. A Implantação de Prontuário Eletrônico em Unidade de Pronto-Socorro. Anais do Congresso da Sociedade Brasileira de Informática; 2006.

16. Negreiros MM, Tavares-Neto J. Proposta de prontuário para as equipes de Saúde da Família. Revista APS 2005;8(2):123-42.

17. Murahovsci D. Implantação de prontuário eletrônico em um hospital de grande porte: estudo de caso. [Dissertação]. São Paulo: EAESPIFGV; 2000.

18. Namorato L, Neto AJC, Garani FV, Braga PO, Lustosa SAS. A utilização do prontuário eletrônico do paciente por médicos do Hospital Municipal Dr. Munir Rafful: um estudo de caso. J Health Inform 2013;5(2):39-43.

19. Rodrigues LC, Souza TM, Barbon Jr. S, Lucatto Jr. RV. Gerenciamento de Prontuário Eletrônico de Pacientes em Clínica de Atendimento Fisioterápico do Centro Universitário do Norte Paulista. In: XVIII Congresso Brasileiro de Informática em Saúde; 2006.

20. Gomes MAG, Xavier EC, Souza LMV, Shimazaki ME. Prontuário eletrônico odontológico - Curitiba avançando na saúde bucal. [citado 2014 Jul 20]. Disponível em: URL: http://telemedicina.unifesp.br/pub/SBIS/ CBIS2002/dados/arquivos/451.pdf.

21. Sancho LG, Rivers N, Reis GA, Cirino MGW, Sena E. Avaliação do projeto "Gestão Saúde em Rede": um primeiro olhar do período pós-informatização na perspectiva da gestáo. In: Anais do X Congresso Brasileiro de Informática em Saúde; 2006; Florianópolis, Brasil. Sáo Paulo: Sociedade Brasileira de Informática em Saúde; 2006.

22. Santos AF, Trad R, Santos SF, Dornas Júnior G, Costa RB, Ribeiro CA, Ruas SSM. Avaliação da implantação do Prontuário Eletrônico na Secretaria Municipal de Saúde de Belo Horizonte. In: Anais do XVIII Congresso Brasileiro de Engenharia Biomédica - SBIS. São José dos Campos: UniVap; 2002. 